\title{
MECHANICAL STABILITY STUDY OF CAPTURE CAVITY II AT FERMILAB*
}

\author{
M.W. McGee ${ }^{\#}$ and Y. Pischalnikov \\ Fermi National Accelerator Laboratory, Batavia, IL 60510, U.S.A.
}

\begin{abstract}
Problematic resonant conditions at both $18 \mathrm{~Hz}$ and $180 \mathrm{~Hz}$ were encountered and identified early during the commissioning of Capture Cavity II (CC2) at Fermilab. CC2 consists of an external vacuum vessel and a superconducting high gradient (close to $25 \mathrm{MV} / \mathrm{m}$ ) 9-cell $1.3 \mathrm{GHz}$ niobium cavity, transported from DESY for use in the A0 Photoinjector at Fermilab. An ANSYS modal finite element analysis (FEA) was performed in order to isolate the source of the resonance and directed the effort towards stabilization. Using a fast piezoelectric tuner to excite (or shake) the cavity at different frequencies (from $5 \mathrm{~Hz}$ to $250 \mathrm{~Hz}$ ) at a low-range sweep for analysis purposes. Both warm $(300 \mathrm{~K})$ and cold $(1.8 \mathrm{~K})$ accelerometer measurements at the cavity were taken as the resonant "fix" was applied. FEA results, cultural and technical noise investigation, and stabilization techniques are discussed.
\end{abstract}

\section{Introduction}

The so called "dressed cavity" (helium cryostat and cavity combination) was originally designed by CEASaclay, subsequently fabricated at the Deutsches Elektron-Synchrotron (DESY) Laboratory in Hamburg, Germany. The first generation dressed cavity arrived at Fermilab in November 2004. After arrival the first generation cavity was removed from the cryostat. In July 2005, a superconducting RF high gradient cavity DESYMKS “AC68” brought from DESY replaced the original cavity for 2-Kelvin operation in CC2 at ILCTA-Meson Building, Fermilab. This modified cryostat was commissioned at Meson and earmarked for subsequent use at the A0 lab building in conjunction with the A0 Photo Injector.

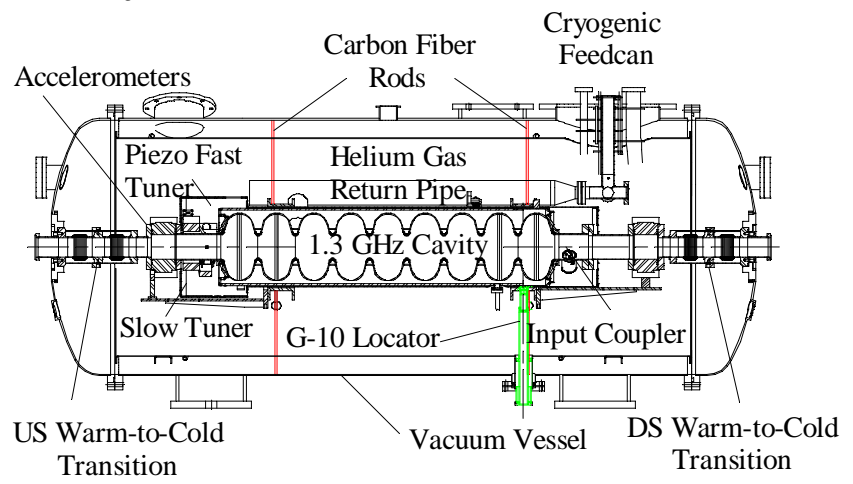

Figure 1: Elevation view of CC2
The elliptical nine-cell cavity was constructed from pure niobium with a specified pre-processed cell wall thickness of $2.8 \mathrm{~mm}$. The end cones which connect the cavity to the helium shell are constructed from $45 \%$ Niobium and $55 \%$ Titanium. A $5 \mathrm{~mm}$ thick helium shell with attached $0.2 \mathrm{~mm}$ thick bellows which exists between the helium shell and upstream (US) end cone allows axial movement to tune the RF cavity. Both the helium and bellows are constructed from Grade 1 Titanium [1].

Modifications to the internal cavity beamline assembly (consisting of a cavity with helium shell, magnetic shield, input coupler, slow and fast tuner) involved the addition of two beam valves cantilevered from the cavity assembly at each end for isolation. This provision was necessary due to several planned transitions for cavity AC-68. A slight warm-to-cold transition design modification was applied due to the additions of these beam valves.

Four tensioned, $10 \mathrm{~mm}$ diameter carbon fiber rods (depicted in red, Figure 1) extend radially from the outer vacuum vessel shell suspending the cavity. While monitoring with perpendicular strain gauges, each carbon fiber rod was tensioned using a turn-buckle type design. The original design from CEA-Saclay involved an individual rod tension of $200 \mathrm{~N}$. In addition, a $28 \mathrm{~mm}$ diameter (G-10) rod (shown in green, Figure 1) locates the longitudinal and transverse position of the cavity with respect to the vacuum shell. Warm-to-cold transitions found US and downstream (DS), limit the flow of ambient heat to the cavity due to thermal conduction. These vacuum vessel to cavity connections both couple the cavity to the outside world and determines the system fundamental frequencies. Also, the input coupler is another spring-type conductive path into the cavity, acting in the transverse direction.

\section{Cryogenic Operation}

Liquid nitrogen and liquid helium are provided to ILCTA-Meson Building through insulated transfer lines from the adjacent Cryogenic Test Facility (CTF). A parallel configuration of three Tevatron satellite refrigerators (with a $625 \mathrm{~W}$ capacity per satellite) provides $4 \mathrm{~g} / \mathrm{s}$ of liquid helium at $4.2 \mathrm{~K}$. Fortunately, many of the mechanical resonance generated by compressors, water skids, vacuum pumps, and expansion engines are limited to CTF, as slight transmission into ILCTA Meson Building occurs. During $1.8 \mathrm{~K}$ operation, a superfluid helium bath was established using a modified liquid ring and root blower vacuum pump system. Also, a LCW water skid is present which creates local technical noise.

\footnotetext{
* Operated by Fermi Research Alliance, LLC, under Contract No. DE-AC0207CH11359 with the U.S. Department of Energy.

\# mcgee@fnal.gov
} 
RF operation at Meson began at $4.2 \mathrm{~K}$ (bathed in liquid helium at atmospheric pressure), although this operating condition was found to be unstable. Nevertheless, a maximum flat top gradient of $15 \mathrm{MV} / \mathrm{m}$ was achieved after applying RF power. Cold operation progressed quickly to superfluid helium temperature as the helium bath was pumped down to 20 torr using the vacuum pump system. Finally, it was possible to achieve a low level radio frequency (LLRF) system with a peak gradient of $31.3 \mathrm{MV} / \mathrm{m}[2]$.

\section{MECHANICAL RESONANCE}

External connections pose a conduit for the propagation of both technical and cultural noise into the cavity. In this case, technical noise has two components; mechanical vibration from the operation of recuperating equipment and thermal acoustic (or pressure) disturbances imposed through the flow of cryogens. The current investigation addresses the first, transmission of mechanical vibration. Flow induced vibration has also affected CC2 operation; however future planned studies will address this issue.

Superconducting RF cavities are sensitive to microphonics (or mechanical disturbances, from an internal or external source) and Lorentz forces (and therefore Lorentz detuning). Several mechanical resonances were measured during operation within the cryostat with very small external excitation present from technical and cultural noise.

Fast piezo tuners provide compensation for both Lorentz detuning and mechanical resonant conditions on some level and frequency range. The piezo driver (used as a sensor) active in the longitudinal direction, combined with two accelerometers (one vertical and one longitudinal) were used to evaluate the microphonic response of the cavity both warm and cold. The two accelerometers, Endevco model 2272 (with sensitivity of $\sim 13 \mathrm{pC} / \mathrm{g}$ ) experienced a factor of three loss in sensitivity, after operating from $300 \mathrm{~K}$ down to $1.8 \mathrm{~K}$. These accelerometer devices were installed under a dual function: to evaluate both the cold accelerometer behavior and mechanical response of the cavity operating at $1.8 \mathrm{~K}$.

RF operation of CC2 was limited by large pulse-topulse amplitudes and phase fluctuations. Most notable was a $188 \mathrm{~Hz}$ resonance excited by RF pulsing, with a small amplitude peak at $168 \mathrm{~Hz}$. A short band of frequencies (within $10 \%$ ) of the major $188 \mathrm{~Hz}$ peak were excited by the same RF pulse [3].

\section{FEA ANALYSIS}

FEA modal studies in ANSYS v10 [4] were conducted in order to understand inherit mechanical resonance present. Component Mode Synthesis (CMS) [5] was applied and represented in Figure 2 consisted of two superelements; the vacuum vessel and cavity assembly.

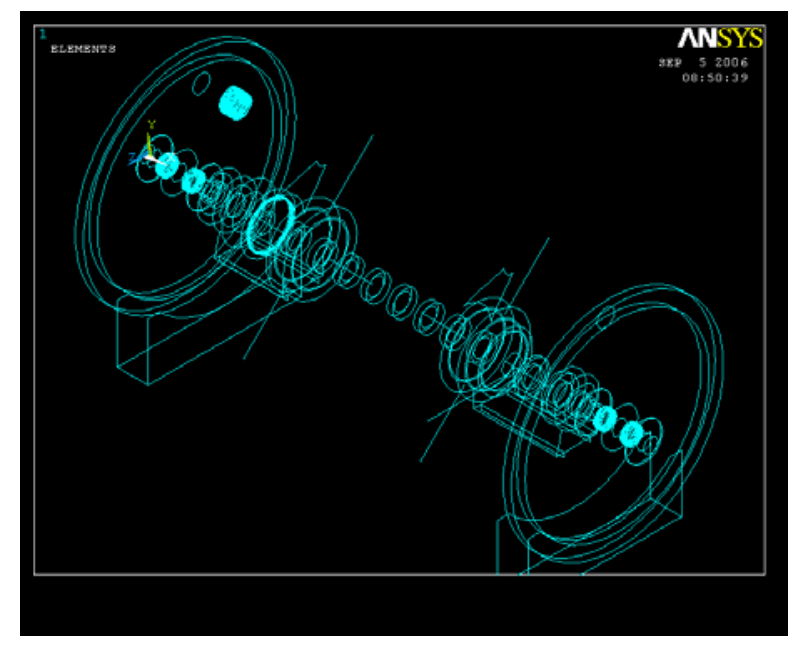

Figure 2: ANSYS CMS representation of CC2 cryostat.

Initially, the modal analysis of the cryostat revealed vertical responses at $18.4 \mathrm{~Hz}$ (shown in Figure 3) and 177 $\mathrm{Hz}$ (shown in Figure 4). At both of these fundamental frequencies, movement at the warm-to-cold transitions was observed.

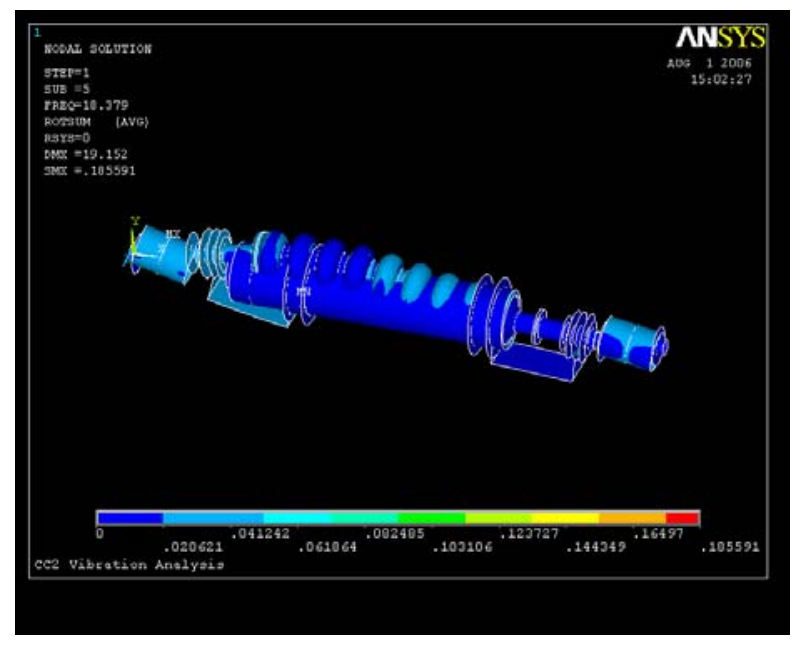

Figure 3: ANSYS modal shape response at $18.4 \mathrm{~Hz}$.

In modal analysis, the reported displacements do not reflect a true estimated value; rather they can indicate relative (or scaled) magnitude of a structure's response at a given frequency. Therefore, the modal results focus on the fundamental frequencies and associated mode shapes. By matching the measured suspect frequencies (with modal shape) to our ANSYS model, we were directed to the possible source of resonance. Since this response can be coupled with other system components it may be difficult to identify the cause and effect. From the measurements (within and outside the cryostat), we learned that the suspect components (warm-to-cold transitions) could be excited by external technical and cultural noise (at $18.4 \mathrm{~Hz}$ ), and RF pulse operation (at 177 $\mathrm{Hz})$. 


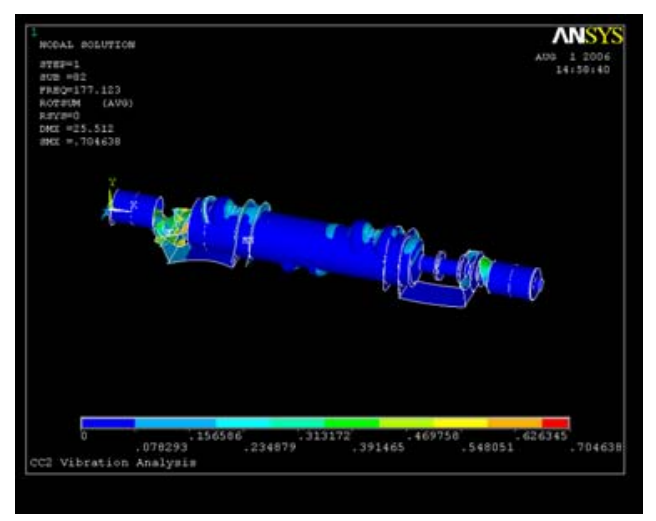

Figure 4: ANSYS modal shape response at $177 \mathrm{~Hz}$.

\section{DESIGN MODIFICATION AND MEASUREMENT}

Through FEA analysis, the warm-to-cold transitions were identified as a major source of mechanical resonance. As a result, both US and DS transitions were modified with a G-10 radial constraint design to limit transmission of vibration through the soft spring-like bellows system. In the original configuration, the warmto-cold transition acted as a classical spring-mass-spring system. The modification held the center mass, since left unconstrained, modes at $18 \mathrm{~Hz}$ will allow external noise to be transmitted within and modes at $180 \mathrm{~Hz}$ are excited by RF operation internally.

\section{Continuous Wave (CW) Mode Operation}

The addition to the slow tuner of a fast acting tuner is required during high gradient pulsed operation to control the cavity frequency. Conversely, CW operation (with a high external Q-value) is a method to measure mechanical perturbations and phase shift between forward power and field probe signals. During CW mode, microphonics (or the influence of coldmass vibration on cavity operation) has the potential to induce large phase and amplitude oscillations in accelerating cavity voltage [5].

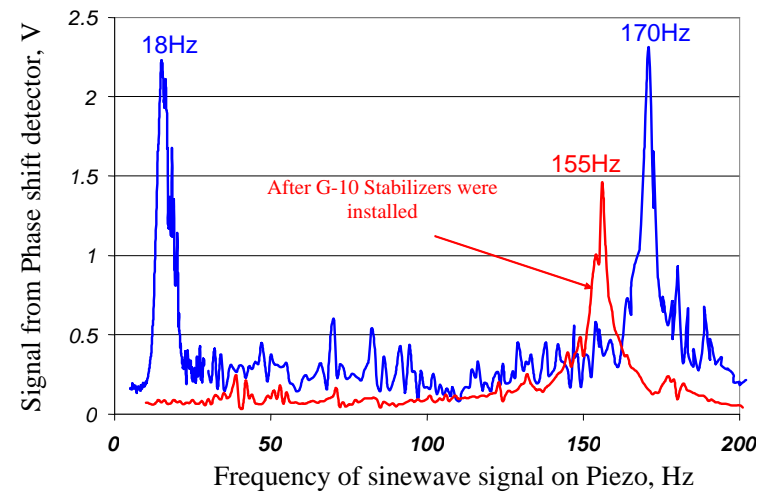

Figure 5: CC2 mechanical transfer function before and after installation of G-10 stabilizer.

\section{Piezo-electric Excitation}

Figure 5 shows the measurements of the transfer function before and after the G-10 constraint installation. Driven with a sine-wave signal, the fast piezo tuner mechanically excites the cavity. A $1.5 \mathrm{~V}$ amplitude piezo driver signal moved the tuner $\sim 60 \mathrm{~nm}$ at $1.8 \mathrm{~K}$. The fast piezo tuner swept from a frequency of $5 \mathrm{~Hz}$ to $250 \mathrm{~Hz}$ with an increment of $\Delta F=1 \mathrm{~Hz}$ as the CC2 transfer function developed. Signal from phase shift detector has been recorded and analyzed to measure reaction of cavity on piezo-induced vibrations.

\section{FUTURE WORK}

After the modification and CC2 operation had been regained, another vibration issue surfaced under different operating modes related to liquid level inventory and supply pressure. Large vertical oscillations were found at $20 \mathrm{~Hz}$ and below [6, 7]. Future tests using CC2 will provide insight regarding the flow induced vibration conditions manifested by pressure variation of helium single-phase supply.

\section{ACKNOWLEDGEMENTS}

Special thanks to the CC2 Managers: Helen Edwards, Ray Fliller, Tim Koeth, Elvin Harms and Andy Hocker for their advice and support. Expert cryogenic support was provided by Accelerator Division Cryogenics Department: Christine Darve, Arkadiy Klebaner and Bill Soyars.

\section{REFERENCES}

[1] M. McGee, "DESY Superconducting Radio Frequency Capture Cavity II 'RSB 569',’ FNAL Pressure Vessel Engineering Note, 2005.

[2] T. Koeth et al., "Construction, Commissioning and A Test Cryogenic Performances of the First TESL Facility (TTF) Cryomodule”, Advances in Cryogenic Engineering, Vol 43, 1998, p. 87. 2006.

[3] www.ANSYS.com

[4] U. Sellgren, "COMPONENT MODE SYNTHESIS A method for efficient dynamic simulation of complex technical systems", VISP WP5 Document Data, Stockholm, 2003.

[5] C. Pagani and P. Pierini, "Cryomodule Design, Assembly and Alignment”, SUP’04, 2004.

[6] C. Darve, private communication, 2007.

[7] Y. Pischalnikov, et al., "A technique for Monitoring Fast Tuner Piezoactuator Preload Forces for Superconducting RF Cavities”, PAC'07, also in these proceedings, 2007. 